\title{
FOUR ALGEBRAIC SPECTRAL PROPERTIES
}

\author{
FREDERICK W. CALL
}

\begin{abstract}
For a commutative ring, we examine the four concepts of flat surjections, exact torsion functors, induced closed affine subschemes, and type I BSP torsion theories. We show they are equivalent, they correspond to closed subsets of Spec that are also stable under generalization, and thus are spectral in nature.
\end{abstract}

Introduction. In the literature there occur four concepts for a commutative ring which this note will show are equivalent. They are $(1)[\mathbf{4}, \mathbf{5}]$ flat surjection, i.e., the ring homomorphism $\phi: A \rightarrow A / I$ where $A / I$ is flat as an $A$-module, (2) [4] a closed subset $Y \subseteq X=\operatorname{Spec} A$ such that the sheaf induced on $Y$ from the canonical sheaf $\tilde{A}$ on $X$ yields an affine scheme, (3) [2] a torsion class $\tau$ that is of type I BSP (the Bounded Splitting Property), and (4) [3] an exact torsion functor $\tau$ on the category of $A$-modules. (We shall use $\tau$ for the torsion class, the torsion radical, and the corresponding subfunctor of the identity functor.) We show they are spectral properties by relating them to the closed subsets $Y$ of $\operatorname{Spec} A$ that are stable under generalization (generically closed).

The correspondences. First we recall some torsion theory terminology [5]. Let $\tau$ be a torsion class, an isomorphism class of $A$-modules closed under submodules, direct sums, extensions, and homomorphic images. The torsion radical $\tau$ is defined by the torsion submodule $T(M)$ of an $A$-module $M$, the largest submodule of $M$ in the class $\tau$. This also defines a subfunctor $\tau$ of the identity which is left exact. The torsion class (radical, functor) $\tau$ is called half-centered $[\mathbf{1}]$ when it is uniquely determined by a set $G$ of prime ideals, closed under generalization, by $\tau=\tau_{G}=\left\{M \in A\right.$-mod $\left.\mid \forall p \in G, M_{p}=0\right\}$. The filter $F(\tau)=\{I \subseteq A \mid A / I \in \tau\}$ determines the class; thus a half-centered theory $\tau$ is also determined by the set $F(\tau) \cap X=X \backslash G$, the set of torsion primes. A filter is the filter of a half-centered theory if it contains a cofinal subset of finitely generated ideals [5, Corollary VI, 6.15]. If the filter $F(\tau)$ contains a cofinal subset of principal ideals, then $\tau(M)$ is just the kernel of the localization $M \rightarrow S^{-1} M$ where $S=\{s \in A \mid s A \in F(\tau)\}$. A special case of this is the $T$-comaximal theory of $[2, \S 1]$ where $T$ is an ideal of $A$ and $F(\tau)=\{I \subseteq A \mid I+T=A\}$. In this case, $S=1+T$. The type I BSP theory of $[2, \S 3]$ is just a $\tau(A)$-comaximal theory, and there they show that

Proposition 1. There is a 1-1 correspondence between type I BSP theories, and ideals $T \subseteq A$ such that the surjection $A \rightarrow A / T$ is flat.

Received by the editors June 18, 1985 and, in revised form, November 19, 1985.

1980 Mathematics Subject Classification (1985 Revision). Primary 13D30, 13C11, 13C12, 14 E40.

Key words and phrases. Flat surjection, torsion theory, affine scheme, exact torsion functor, flat epimorphism. 
They also remark that there is a 1-1 correspondence between these theories and hereditary TTF-classes. See [3, Proposition I 5.5: (4) $\Leftrightarrow(5)]$ for a more detailed proof of this latter fact.

We use $V(T)$ for the set of prime ideals of $A$ containing $T$. For a ring homomorphism $\phi: A \rightarrow B$, we denote by ${ }^{a} \phi: \operatorname{Spec} B \rightarrow \operatorname{Spec} A$ the continuous map defined by ${ }^{a} \phi(p)=\phi^{-1}(p)$.

LEMMA 2. The generic closure of a closed subset of $\operatorname{Spec} A$ is affine.

Proof. Let $V=V(T)$ be closed $\subseteq X, W=\{q \in \operatorname{Spec} A \mid \exists p \in V, p \supseteq$ $q$ \}. If $S=1+T$ then the map $\phi: A \rightarrow S^{-1} A$ yields the homeomorphism ${ }^{a} \phi: \operatorname{Spec} S^{-1} A \rightarrow W \subseteq \operatorname{Spec} A$.

Proposition 3. The following are equivalent for an ideal $T \subseteq A$ :

(a) the projection $A \rightarrow A / T$ is flat. $T$.

(b) the natural map $\phi: A \rightarrow S^{-1} A$ is a surjection where $S=1+T$, and $\operatorname{ker} \phi=$

(c) $T$ is a type I BSP theory and $T=\tau(A)$.

(d) $V$ is a closed subset of $\operatorname{Spec} A$, stable under generalization, and $T$ is the least element in the set $\left\{T^{\prime} \subseteq A \mid V\left(T^{\prime}\right)=V\right\}$.

ProOF. The equivalence of (a) and (c) has been discussed and (b) $\Rightarrow(\mathrm{a})$ is trivial. (a) $\Rightarrow(\mathrm{d})$. If $A / T$ is flat then, for any $p \in X, A_{p} / T_{p}$ is a free $A_{p}$-module. Hence $T_{p}=0$ or $T_{p}=A_{p}$, the latter holding if and only if $p \notin V(T)$. Thus, for each $p \in V(T), A_{p} \cong(A / T)_{p}$. This yields that $V(T)$ is generically closed. If $p \in V\left(T^{\prime}\right)$ then $0=T_{p} \subseteq T_{p}^{\prime}$, while if $p \notin V\left(T^{\prime}\right)$ then $T_{p}=A_{p}=T_{p}^{\prime}$. In either case, $T \subseteq T^{\prime}$, and $T$ is least.

(d) $\Rightarrow(\mathrm{b})$. Let $S=\{s \in A \mid V(s A) \cap V=\varnothing\}$. If $p \in V$, then $A_{p} \rightarrow\left(S^{-1} A\right)_{p}$ is a further localization, hence an isomorphism. If $p \notin V$ then $\left(S^{-1} A\right)_{p}=0$ since $\operatorname{Spec}\left(S^{-1} A\right)_{p}=\operatorname{Spec} S^{-1} A \cap \operatorname{Spec} A_{p}=\varnothing$. From these facts we conclude that $\phi: A \rightarrow S^{-1} A$ is a surjection, and, if $K=\operatorname{ker} \phi, 0=K_{p} \subseteq T_{p}$ for all $p \in V$, while $K_{p}=A_{p}=T_{p}$ if $p \notin V$. Thus $K \subseteq T$ and $V(K)=V$. Since $T$ is least, $K=T$. Clearly, we may replace $S$ by $1+T$.

COROLlaRY 4. There is a 1-1 correspondence between closed subsets of Spec $A$ stable under generalization, and ideals $T \subseteq A$ such that $A / T$ is flat.

PROOF. For our correspondence, we map $T$, if $A / T$ is flat, to $V(T)$ which is closed under generalization. From the proof $(\mathrm{d}) \Rightarrow(\mathrm{b})$, if $V$ is a closed set closed under generalization and $S=\{s \in A \mid V(s A) \cap V=\varnothing\}$ and $K=\operatorname{ker}\left(A \rightarrow S^{-1} A\right)$, then $A / K=S^{-1} A$ is flat and $V(K)=V$. Thus our map is onto. If $A / T_{1}$ and $A / T_{2}$ are flat and $V\left(T_{1}\right)=V\left(T_{2}\right)$, then $T_{1}=T_{2}$ by $(\mathrm{a}) \Rightarrow(\mathrm{d})$, so the correspondence is $1-1$.

We now examine when a torsion functor $\tau$ is (right) exact.

Proposition 5. A torsion functor $\tau$ is exact if and only if $\tau=\tau_{G}$ for some closed subset $G \subseteq \operatorname{Spec} A$ that is stable under generalization. This establishes a 1-1 correspondence between exact torsion functors on $A$-modules and closed subsets of Spec $A$, stable under generalization.

ProOF. In [3, Proposition I 5.5: (1) $\Leftrightarrow(5)]$ it is shown that $\tau$ is exact if and only if $F(\tau)=\{I \mid I+\tau(A)=A\}$. Such a filter, we have seen, has a cofinal 
subset of principal ideals. Thus $\tau=\tau_{G}$, where $G=\{p \in X \mid p+\tau(A) \neq$ $A\}=V(\tau(A))$, since $\tau(A) \subseteq \bigcap G$. Hence $G$ is closed and generically closed. Conversely, if $V \subseteq$ Spec $A$ is closed and generically closed, then from the proof of Proposition $1(\mathrm{~d}) \Rightarrow(\mathrm{b}), F\left(\tau_{V}\right)=\{I \mid I+K=A\}, K=\operatorname{ker}\left(A \rightarrow S^{-1} A\right)$ where $S=\{s \in A \mid V(s A) \cap V=\varnothing\}$. Clearly, $K=\tau_{V}(A)$. Hence by $[\mathbf{3}], \tau_{V}$ is exact, and $V\left(\tau_{V}(A)\right)=V(K)=V$. Finally, we note that $\tau_{G_{1}}=\tau_{G_{2}}$ if and only if $G_{1}=G_{2}$, when $G_{1}$ and $G_{2}$ are generically closed.

If $Y \subseteq X=\operatorname{Spec} A$, and $\tilde{A}$ is the canonical sheaf on $X, Y$ is called an induced affine subscheme or an Affine subscheme or even just Affine if the scheme $\left(Y, i^{-1} \tilde{A}\right)$ is an affine scheme ( $i$ is the inclusion map). In [4, Proposition IV, 2.5], it is shown that each Affine $Y \subseteq \operatorname{Spec} A$ is stable under generalization, is quasi-compact, and corresponds to (the isomorphism class of) a flat epimorphism $\phi: A \rightarrow B$. In this correspondence $Y$ is homeomorphic to $\operatorname{Spec} B$ by the induced map ${ }^{a} \phi: \operatorname{Spec} B \rightarrow$ Spec $A$. If we identify $Y$ with $\operatorname{Spec} B$, then $i^{-1} \tilde{A}=\tilde{B}$.

The next proposition follows from this and Lemma 2, Proposition 3, and Corollary 4.

Proposition 6. A closed subset $V \subseteq \operatorname{Spec} A$ is stable under generalization if and only if it is Affine. In this case there is a least element $T$ in the set $\left\{T^{\prime}\right.$ $\left.V\left(T^{\prime}\right)=V\right\}$, and the induced sheaf $i^{-1} \tilde{A}$ on $V$ is just $\widetilde{A / T}$.

REMARKS 7. The closed Affine subsets of $\operatorname{Spec} A$ are trivial (two kinds) for domains and local rings. A noetherian ring $A$ has only finitely many closed Affines, since $A$ can be decomposed into a finite product $\prod A_{i}$ so that each closed Affine of $\operatorname{Spec} A$ is trivial on $\operatorname{Spec} A_{i}$. For zero dimensional rings, closed, compact, and Affine are the same.

ACKNOWLEDGMENT. The author thanks the University of Sheffield for the use of their facilities in the preparation of this paper while he was a visitor.

\section{REFERENCES}

1. P.-J. Cahen, Torsion theory and associated primes, Proc. Amer. Math. Soc. 38 (1973), 471-476.

2. F. W. Call and T. S. Shores, The splitting of bounded torsion submodules, Comm. Algebra 9(11) (1981), 1161-1214.

3. J. S. Golan, Localization of non-commutative rings, Pure and Appl. Math., Marcel Dekker, New York, 1975.

4. D. Lazard, Autour de la platitude, Bull. Soc. Math. France 97 (1969), 81-128.

5. B. Stenström, Rings of quotients, Grundlehren Math. Wiss., vol. 217, Springer-Verlag, New York, Heidelberg and Berlin, 1975.

Department of Pure Mathematics, University of Sheffield, Sheffield S3 7RH, ENGLAND

Current address: Department of Mathematical Sciences, University of Wisconsin-Milwaukee, P. O. Box 413, Milwaukee, Wisconsin 53201 